\title{
Fungurie nei pazienti ospedalizzati: indagine retrospettiva multicentrica
}

\author{
Elisabetta Faggi',2, Claudio Farina ${ }^{1,3}$, Esther Manso ${ }^{1,4}$, Stefano Andreoni, ${ }^{1,5}$, Gianluigi Lombardi, \\ Paolo Fazii ${ }^{1,7}$, Gabriella Pini ${ }^{2}$, Gioconda Brigante ${ }^{6}$, Pierluigi Nicoletti ${ }^{8}$, Giorgio Verna $^{4}$ \\ 'Comitato Studio Micologia (CoSM), Associazione Microbiologi Clinici Italiani (AMCLI) \\ ${ }^{2}$ Dipartimento Sanità Pubblica, sez. Microbiologia - Università di Firenze \\ ${ }^{3}$ A.O. Ospedali Riuniti di Bergamo - Bergamo - \\ ${ }^{4}$ A.O. Umberto I - Ancona \\ ${ }^{5}$ A.O. Ospedale Maggiore della Carità - Novara \\ ${ }^{6}$ Ospedale di Circolo e Università dell'Insubria - Varese \\ ${ }^{7}$ P.O. Ospedale Spirito Santo - Pescara \\ ${ }^{8}$ A.O. Careggi - Firenze \\ - Indirizzo attuale: AO ‘Ospedale San Carlo Borromeo, Milano
}

Key words: Funguria, Fungemia, Candida

Funguria in hospitalized patients: multicenter retrospective survey.

\section{SUMMARY}

A multicenter retrospective survey of funguria was run at 6 Italian hospitals (Bergamo, Novara,Varese, Florence, Ancona, Pescara) from January I, 200 I to December 3I, 2002. The aim of the study was to evaluate the incidence of recovery of yeasts from urine cultures, the distribution among the hospital wards, the involved species and the number of patients with concurrent fungemia and funguria.

Microorganisms (either bacteria or yeasts) were isolated from the $21 \%$ of urine cultures: overall, $2 \%$ of them were positive for yeasts, whereas $19 \%$ for bacteria. Yeasts were recovered from the $8 \%$ of the positive urine cultures.

Yeasts in the urine were mostly observed in Intensive Care Units (24\% of positive urine cultures), and less frequently in Medical and Surgical wards.

Candida albicans was the most frequently recovered species (63\%), followed by C. glabrata (18\%), C. tropicalis (9\%), C. parapsilosis (3\%); other Candida species, Trichosporon asahii and Saccharomyces cerevisiae were occasionally isolated, whereas moulds were never recovered.

Overall, $5 \%$ of patients (55/1 II9) with funguria had concurrent fungemia and in 41 cases the same species was recovered from both urine and blood. $C$. albicans was the most frequently recovered species, followed by $C$. glabrata, C. tropicalis and C. parapsilosis.

\section{INTRODUZIONE E SCOPO DELLA RICERCA}

L'isolamento di funghi dalle urine è un evento infrequente nella popolazione generale sia adulta che pediatrica (3) mentre numerose sono le segnalazioni nella popolazione ospedalizzata, in particolare in quella ricoverata in reparti di Terapia Intensiva $(7,10,18)$.

Lieviti appartenenti al genere Candida sono quelli più frequentemente ritrovati; saltuariamente sono stati isolati altri lieviti (Trichosporon, Cryptococcus, Saccharomyces ecc), eccezionalmente funghi filamentosi (Aspergillus, Fusarium) $(9,12,16)$.

L'interpretazione da dare all'isolamento di miceti dalle urine è non facile, in particolare per quello di lieviti appartenenti al genere Candida. Il loro isolamento, infatti, potrebbe essere dovuto ad una contaminazione da parte della flora microbica perineale, ad una colonizzazione transitoria del distretto urinario o ad una vera e propria infezione conseguente o meno ad una candidemia.

Lo scopo dell'indagine che il Comitato Studio Micologia (CoSM) dell'AMCLI ha proposto, è stato quello di valutare, con un'indagine retrospettiva multicentrica sulle fungurie in ambiente ospedaliero, gli aspetti epidemiologici di tale condizione in relazione alle batteriurie ed alle fungemie.

\section{MATERIALI E METODI}

L'indagine è stata condotta in sei centri ospedalieri italiani (Bergamo, Novara, Varese, Firenze, Ancona, Pescara) nel periodo 1 gennaio 2001 31 dicembre 2002.

Sono stati valutati retrospettivamente, a partire dai dati degli archivi informatici dei laboratori di Microbiologia, i risultati di 101024 urinocolture provenienti da pazienti ricoverati in reparti di Terapia Intensiva, in reparti chirurgici ed in repar- 
ti medici. Sono state considerate positive soltanto le urinocolture con carica microbica (fungina $o$ batterica, con esclusione delle batteriurie polimicrobiche) $\geqslant 10^{4} \mathrm{UFC} / \mathrm{ml}$.

Queste sono state suddivise in base ai reparti e alla modalità di prelievo (catetere vescicale, mitto intermedio ecc).

La distribuzione delle varie specie fungine è stata effettuata sia in relazione al numero di urinocolture che al numero dei pazienti. In pazienti con funguria, oltre all'età e al sesso, è stata indagata l'eventuale presenza di fungemia (insorta contemporaneamente alla funguria) ed i relativi agenti eziologici.

\section{RISULTATI}

Il $21,71 \%$ delle urinocolture (su un totale di 101024 ) presentò un carica microbica $\geqslant 10^{4}$ UFC/ml: nell' $1,7 \%$ l'eziologia fu fungina, nel $19,01 \%$ batterica (tabella 1 ).

Miceti furono isolati nell' $8,2 \%$ delle urinocolture positive (tabella 2).

Nelle urinicolture positive, le fungurie sono state significativamente più frequenti $(p<0,001)$ nei reparti di Terapia Intensiva rispetto ai reparti medici e chirurgici (tabella 3 )

L' $80 \%$ delle urine positive per miceti è stato prelevato da catetere vescicale, $1 \%$ da catetere nefrostomico, il 19\% da mitto intermedio.

Tali urine provenivano da 1119 pazienti prevalentemente di sesso femminile [677 (60,5\%) femmine, $442(39,5 \%)$ maschi] e con una età media di 67 anni $\pm 18,32$.

La frequenza di isolamento delle varie specie fungine è simile sia se vengono prese in considerazione il numero delle urinocolture positive per miceti sia il numero dei pazienti con funguria (tabella 4).

Candida albicans è la specie maggiormente isolata, seguita da C. glabrata, C. tropicalis, C. parapsilosis; saltuariamente sono state isolate altre specie di Candida, Trichosporon asahii e Saccharomyces cerevisiae.

In alcuni pazienti sono state isolate più specie dalla stessa urinocoltura o da urinocolture effettuate in tempi diversi (le associazioni più frequente furono: $C$. albicans-C. glabrata, $C$. albicans-C. parapsilosis e C. glabrata-C. tropicalis). Non sono mai stati ritrovati funghi filamentosi. Il 5\% dei pazienti con funguria presentò fungemia (55 pazienti su 1119): la fungemia fu causata dalla stessa specie fungina della funguria in 41 pazienti, in 14 invece da specie diverse. In tutti questi pazienti furono isolati soltanto lieviti appartenenti al genere Candida.

Le fungemie associate a fungurie (causate dalla stessa specie) si osservarono soprattutto nei repar- ti chirurgici e di Terapia Intensiva (tabella 5) e la specie maggiormente isolata fu C. albicans seguita da C. glabrata, C. tropicalis, C. parapsilosis (tabella 6).

Non furono notate differenze significative fra la carica micotica urinaria ritrovata in pazienti con funguria senza fungemia e quella ritrovata in pazienti con funguria e fungemia causata dalla stessa specie fungina (tabella 7).

Tabella I. Frequenza di isolamento di miceti e batteri nelle urinocolture di pazienti ospedalizzati

\begin{tabular}{lc}
\hline & $\mathrm{N}^{\circ}$ urinocolture (\%) \\
\hline Urinocolture positive per miceti & $1716(1,70)$ \\
\hline Urinocolture positive per batteri & $19207(19,01)$ \\
\hline Urinocolture negative & $80101 \quad(79,29)$ \\
\hline Urinocolture totali & $101024(100)$ \\
\hline
\end{tabular}

Tabella 2. Frequenza di isolamento di miceti e batteri nelle urinocolture positive

\begin{tabular}{lc}
\hline & $N^{\circ}$ urinocolture (\%) \\
\hline Urinocolture positive per miceti & $1716(8,20)$ \\
\hline Urinocolture positive per batteri & $19207(91,80)$ \\
\hline Totale urinocolture positive & $20923(100)$ \\
\hline
\end{tabular}

Tabella 3. Prevalenza di fungurie nelle urinocolture positive: suddivisione in base al tipo di reparto*

\begin{tabular}{llc}
\hline \multicolumn{1}{c}{ urinocolture positive } & $\begin{array}{c}\mathrm{N}^{\circ} \text { urinocolture } \\
\text { positive per miceti (\%) }\end{array}$ \\
$\begin{array}{llc}\text { Reparti di Terapia } \\
\text { Intensiva }\end{array}$ & 1507 & $365(24,22)$ \\
\hline Reparti medici & 8859 & $469(5,29)$ \\
\hline Reparti chirurgici & 3377 & $245(7,25)$ \\
\hline *Casistica di 4 centri ospedalieri; per 2 centri non è stato possi- \\
bile stratificare le batteriurie per reparto
\end{tabular}

Tabella 4. Agenti eziologici delle fungurie suddivisi per urinocolture e per paziente

\begin{tabular}{|c|c|c|c|c|}
\hline \multicolumn{2}{|c|}{$\mathrm{N}^{\circ}$ urinocolture } & $\%$ & $\mathrm{~N}^{\circ}$ pazienti & $\%$ \\
\hline C. albicans & 1081 & 63,00 & 725 & 64,79 \\
\hline C. tropicalis & 154 & 8,97 & 98 & 8,76 \\
\hline C. krusei & 34 & 1,98 & 22 & 1,97 \\
\hline C. glabrata & 301 & 17,54 & 177 & 15,82 \\
\hline C. parapsilosis & 55 & 3,21 & 36 & 3,22 \\
\hline C. lusitaniae & 30 & 1,75 & 18 & $1,6 I$ \\
\hline C. guilliermondii & 3 & 0,17 & 3 & 0,27 \\
\hline C. kefyr & 6 & 0,35 & 5 & 0,45 \\
\hline C. dubliniensis & 2 & 0,12 & 2 & 0,18 \\
\hline C. inconspicua & 2 & 0,12 & 2 & 0,18 \\
\hline C. pelliculosa & 4 & 0,23 & 3 & 0,27 \\
\hline C. catenulata & I & 0,06 & $\mathrm{I}$ & 0,09 \\
\hline Candida spp & 32 & 1,86 & 15 & 1,34 \\
\hline S. cerevisiae & $\mathrm{I}$ & 0,06 & $\mathrm{I}$ & 0,09 \\
\hline T. asahii & 3 & 0,17 & 2 & 0,18 \\
\hline Più specie & $7 *$ & $0,4 I$ & $9 * *$ & 0,80 \\
\hline Totale & 1716 & 100 & 1119 & 100 \\
\hline
\end{tabular}

*Più specie isolate contemporaneamente

** Più specie isolate contemporaneamente o in tempi diversi dallo stesso paziente 
Tabella 5. Pazienti con funguria e fungemia causata dalla stessa specie: suddivisione in base ai reparti

\begin{tabular}{|c|c|c|}
\hline & $\begin{array}{l}\text { Totale pazienti } \\
\text { con funguria }\end{array}$ & $\mathrm{N}^{\circ}$ pazienti \\
\hline
\end{tabular}

Reparti di Terapia con funguria con funguria e fungemia $(\%$

\begin{tabular}{llll} 
Intensiva & 305 & 13 & $(4,26)$ \\
\hline Reparti medici & 572 & I7 & $(2,97)$ \\
\hline Reparti chirurgici & 242 & II & $(4,55)$ \\
\hline
\end{tabular}

Tabella 6. Agenti eziologici di fungurie e fungemie causate dalla stessa specie

\begin{tabular}{ll}
\hline & $\mathrm{N}^{\circ}$ pazienti $(\%)$ \\
\hline C. albicans & $27(65,85)$ \\
\hline C. glabrata & $6(14,63)$ \\
\hline C. tropicalis & $6(14,63)$ \\
\hline C. parapsilosis & $2(4,88)$ \\
\hline Totale & $4 \mathrm{I}(100)$ \\
\hline
\end{tabular}

Tabella 7. Concentrazione di lieviti nelle urine (UFC/ml) di pazienti con funguria senza fungemia e pazienti con funguria e fungemia*

\begin{tabular}{llll}
\hline & \multicolumn{3}{c}{ Concentrazione di lieviti nelle urine } \\
& $10^{4} \mathrm{UFC} / \mathrm{ml}$ & $\geq 10^{5} \mathrm{UFC} / \mathrm{ml}$ & Totale \\
\hline $\mathrm{N}^{\circ}$ pazienti & & & \\
con funguria & & & \\
senza fungemia & $232(21,52 \%)$ & $846(78,48 \%)$ & $1078(100 \%)$ \\
\hline$N^{\circ}$ pazienti & & & \\
con funguria & & & \\
e fungemia** & $12(29,27 \%)$ & $29(70,73 \%)$ & $41(100 \%)$ \\
\hline
\end{tabular}

*Nei pazienti che hanno effettuato più di una urinocoltura, è stata considerata quella con carica microbica maggiore;

**causata dalla stessa specie fungina

\section{CONCLUSIONI}

Il significato clinico della presenza di lieviti nelle urine è un argomento estremamente dibattuto. Infatti, per alcuni autori cariche fungine $\geqslant 10^{4}$ $\mathrm{UFC} / \mathrm{ml}$ (anche in pazienti con catetere vescicale) hanno un significato clinico e correlano con candidosi del tratto urinario dimostrate all'autopsia $(4,8)$. Anche Chabasse (3) segnala una correlazione significativa fra cariche fungine $>10^{4}$ $\mathrm{UFC} / \mathrm{ml}$ (lieviti isolati da qualsiasi distretto corporeo compreso quello urinario) e candidosi profonda.

Altri Autori $(5,15)$, al contrario, affermano che anche cariche fungine $>10^{4} \mathrm{UFC} / \mathrm{ml}$ non dimostrano la presenza di una candidosi delle vie urinarie né di una candidosi sistemica.

L'esistenza di questa problematica ha stimolato il CoSM ad intraprendere questa ricerca retrospettiva sulle fungurie. La nostra indagine non ha preteso di risolvere i molti interrogativi posti dall'isolamento dei miceti dalle urine ma di valutarne, in una ampia casistica italiana, la frequenza, gli agenti eziologici e l'associazione con fungemie.

I dati da noi raccolti hanno mostrato che il ritro- vamento di miceti nelle urine è infrequente rispetto a quello di batteri. Infatti, soltanto 1' $1,7 \%$ delle urinocolture effettuate risulta positivo per miceti mentre il $19 \%$ lo è per batteri. Frequenze simili furono riportate anche da autori spagnoli (11) che riportano una positività per miceti nell' $1,3 \%$ delle urinocolture totali effettuate presso un centro ospedaliero mentre casistiche americane riportano frequenze più elevate $(5 \%)(13)$. Tuttavia se si considerano i reparti di degenza, abbiamo osservato che le candidurie sono particolarmente frequenti nei reparti di Terapia Intensiva dove rappresentano il $24 \%$ delle urinocolture positive correlando molto bene con la gravità delle patologie dei pazienti ivi ricoverati.

Sebbene $C$. albicans sia la specie più frequentemente ritrovata, le eziologie da $C$. glabrata e $C$. tropicalis rappresentano il 18\% e il 9\% rispettivamente delle fungurie. Le eziologie da Candidae non albicans si sono fatte sempre più frequenti negli ultimi anni $(1,13)$ soprattutto in conseguenza della profilassi e della terapia con triazolici, in particolare fluconazolo, che notoriamente è in grado di indurre resistenze in varie specie di Candida non albicans fra cui, in particolare, $C$. glabrata (6). I nostri dati sono in accordo con altre casistiche che riportano frequenze di $15-36 \%$ per C. glabrata e di 7-15\% per C. tropicalis (2, 7, 14,17 )

La candiduria può essere anche uno dei primi segni di candidosi disseminata in cui i lieviti, in seguito ad una diffusione per via ematica, si possono localizzare a livello renale. È ben noto infatti il tropismo di C. albicans per il rene sia nelle infezioni sperimentali che nelle indagini autoptiche sull'uomo. Tuttavia, in assenza di reperti istologici, non si può escludere che la presenza di lieviti nelle urine, anche in pazienti con candidemia, sia dovuta a colonizzazione o infezione delle basse vie urinarie piuttosto che ad una vera e propria micosi renale.

Nella nostra indagine abbiamo osservato che il $4 \%$ dei pazienti con funguria presentava anche fungemia causata dalla stessa specie. I nostri dati concordano con quelli di altri autori che riportano candidemie nell' 1 - 20\% $(7,15,19)$ dei pazienti con candiduria.

Il fatto che non furono notate differenze significative fra la carica micotica urinaria ritrovata in pazienti con funguria senza fungemia e quella ritrovata in pazienti con funguria e fungemia, ripropone il dilemma diagnostico sul significato da attribuire alla quantità di lieviti nelle urine.

A nostro avviso il problema non può essere risolto soltanto in base alla carica fungina nelle urine ma può esserlo tenendo conto delle caratteristiche cliniche ed epidemiologiche del paziente e della 
contemporanea presenza di molteplici fattori di rischio che possono favorire l'insorgenza di infezioni fungine (terapia antibiotica, diabete, immunosoppressione, catetere venoso, catetere vescicale, ecc). L'unico fattore di rischio che in questa indagine retrospettiva abbiamo potuto accertare è stata la presenza di catetere vescicale $(80 \%$ dei prelievi positivi per miceti): ulteriori ricerche mirate a precisare $\mathrm{i}$ fattori di rischio, la terapia e l'evoluzione micologica potrebbero portare un contributo alla conoscenza dei molteplici problemi posti dall'isolamento dei funghi dalle urine.

\section{BIBLIOGRAFIA}

1. Al-Hedaithy SS, Fotedar R. Prevalence of Candida tropicalis in clinical specimens from patients with variable clinical syndromes over a 5-year period. Mycoses 1997; 40: 111-3

2. Ayeni O, Riederer KM, Wilson FM et al. Clinicians' reaction to positive urine culture for Candida organisms. Mycoses 1999; 42: 285-9

3. Chabasse D. Intérêt de la numération des levures dans les urines. Revue de la littérature et résultats préliminaires d'une enquête multicentrique réalisée dans 15 centres hospitaliers universitaires. Ann Fr Anesth Reanim 2001; 20: 400-6

4. Goldberg PK, Kozinn PJ, Wise GJ et al. Incidence and significance of candiduria. JAMA 1979; 241: $582-4$

5. Haley LD. Yeast infections of the lower urinary tract. Sabouraudia 1965;4: 98-105.

6. Harris AD, Castro J, Sheppard DC et al. Risk factors for nosocomial candiduria due to Candida glabrata and Candida albicans. Clin Infect Dis 1999; 29: 926-8

7. Kauffman CA, Vazquez JA, Sobel JD et al. Prospective multicenter surveillance study of funguria in hospitalized patients. Clin Infect Dis 2000; 30:14-8

8. Kozinn PJ, Taschdjian CL, Kodsi BE et al. Diagnosis of systemic or visceral candidosis. Can Med Assoc J 1982 Jun 15;126(12):1386-90

9. Lopes JO, Oliveira LT, Pinto MS, Klock C. Chronic necrotizing pulmonary aspergillosis with concomitant aspergilluria. Mycoses 1997; 40: 209-11

10. Lundstrom T, Sobel J. Nosocomial candiduria: a review. Clin Infect Dis 2001; 32: 1602-7

11. Munoz P, Gijon P, Alcala L et al. How to manage candiduria. Rev Med Microbiol 1998; 9: 225- 31

12. Nakar C, Livny G, Levy I et al. Mycetoma of the renal pelvis caused by Fusarium species. Pediatr Infect Dis J 2001; 20: 1182-3

13. Occhipinti DJ, Gubbins PO, Schreckenberger P et al. Frequency, pathogenicity and microbiologic outcome of non-Candida albicans candiduria. Eur J Clin Microbiol Infect Dis 1994 Jun;13(6):459-67

14. Odds F.C. Ecology of Candida and Epidemiology of Candidosis. In: Candida and Candidosis. A review and Bibliography. Bailliere Tindall Ed, London 1988; 68-92

15. Rivett AG, Perry JA, Cohen J. Urinary candidiasis: a prospective study in hospital patients Urol Res 1986;14(4):183-6

16. Sobel JD, Vazquez JA. Fungal infections of the urinary tract. World J Urol 1999; 17: 410-4

17. Sobel JD, Kauffman CA, McKinsey D et al. Candiduria: a randomized, double-blind study of treatment with fluconazole and placebo. Clin Infect Dis 2000; 30: 19-24.

18. Sobel JD, Lundstrom T. Management of candiduria. Curr Urol Rep 2001; 2: 321-5

19. Storfer SP, Medoff G, Fraser VJ et al. Candiduria: retrospective review in hospitalized patient. Inf Dis Clin Pract 1994; 3:23-9
Elisabetta Faggi

Dipartimento di Sanità Pubblica

Sez Microbiologia, V.le Morgagni 48, 50134 Firenze

Tel 055-3262443; Fax 055-3262446

E-mail: efaggi @unifi.it 